\title{
A Single Bout of Constant-Load Exercise Test for Estimating the Time Constant of Oxygen Uptake Kinetics in Individuals With Stroke
}

\author{
Kazuaki Oyake, PT, PhD ${ }^{1,2}$, Yasuto Baba, PT, BSc ${ }^{2}$, Yuki Suda, PT, MSc ${ }^{2}$, Jun Murayama, PT, BSc ${ }^{2}$, \\ Ayumi Mochida, PT, BSc ${ }^{2}$, Yuki Ito, PT, BSc ${ }^{2}$, Honoka Abe, PT, BSc ${ }^{2}$, Kunitsugu Kondo, MD, PhD ${ }^{2}$, \\ Yohei Otaka, MD, $\mathrm{PhD}^{2,3}$, Kimito Momose, PT, $\mathrm{PhD}^{1}$ \\ ${ }^{1}$ Department of Physical Therapy, School of Health Sciences, Shinshu University, Nagano; \\ ${ }^{2}$ Department of Rehabilitation Medicine, Tokyo Bay Rehabilitation Hospital, Chiba; \\ ${ }^{3}$ Department of Rehabilitation Medicine I, School of Medicine, Fujita Health University, Aichi, Japan
}

\begin{abstract}
Objective To examine the relationship between the time constant of oxygen uptake kinetics during the onset of exercise $\left(\tau \mathrm{VO}_{2}\right)$ estimated from a single exercise bout and that obtained from three averaged exercise bouts in individuals with stroke.

Methods Twenty participants with stroke performed three bouts of a constant-load pedaling exercise at approximately $80 \%$ of the workload corresponding to the ventilatory threshold to estimate $\tau \mathrm{VO}_{2}$. The $\mathrm{VO}_{2}$ data from the first trial of three bouts were used to estimate $\tau \mathrm{VO}_{2}$ for a single bout. Additionally, data collected from three bouts were ensemble-averaged to obtain $\tau \mathrm{VO}_{2}$ for three averaged bouts as the criterion.

Results There was a very high correlation between $\tau \mathrm{VO}_{2}$ for a single bout (34.8 \pm 14.0 seconds) and $\tau \mathrm{VO}_{2}$ for three averaged bouts $\left(38.5 \pm 13.4\right.$ seconds) $(\mathrm{r}=0.926, \mathrm{p}<0.001)$. However, $\tau \mathrm{VO}_{2}$ for a single bout was smaller than that for three averaged bouts $(\mathrm{p}=0.006)$.

Conclusion $\tau \mathrm{VO}_{2}$ for a single bout could reflect the relative difference in $\tau \mathrm{VO}_{2}$ for three averaged bouts among individuals with stroke. However, it should be noted that $\tau \mathrm{VO}_{2}$ for a single bout may be underestimated compared to $\tau \mathrm{VO}_{2}$ for three averaged bouts.
\end{abstract}

Keywords Cerebrovascular disorders, Exercise test, Oxygen consumption, Rehabilitation

Received April 20, 2021; Revised June 5, 2021; Accepted June 17, 2021; Published online August 30, 2021

Corresponding author: Kimito Momose

Department of Physical Therapy, School of Health Sciences, Shinshu University, 3-1-1 Asahi, Matsumoto, Nagano 390-8621, Japan. Tel: +81-263-373453, Fax: +81-263-37-3453, E-mail: kmomose@shinshu-u.ac.jp

ORCID: Kazuaki Oyake (https://orcid.org/0000-0001-6345-1915); Yasuto Baba (https://orcid.org/0000-0003-2723-4643); Yuki Suda (https://orcid. org/0000-0001-8754-8009); Jun Murayama (https://orcid.org/0000-0002-9636-8584); Ayumi Mochida (https://orcid.org/0000-0003-4928-6567); Yuki Ito (https://orcid.org/0000-0001-9972-4815); Honoka Abe (https://orcid.org/0000-0002-3641-0883); Kunitsugu Kondo (https://orcid.org/0000-00026614-8142); Yohei Otaka (https://orcid.org/0000-0002-6797-2782); Kimito Momose (https://orcid.org/0000-0003-3225-4200).

(c) This is an open-access article distributed under the terms of the Creative Commons Attribution Non-Commercial License (http://creativecommons.org/ licenses/by-nc/4.0) which permits unrestricted noncommercial use, distribution, and reproduction in any medium, provided the original work is properly cited. Copyright $\odot 2021$ by Korean Academy of Rehabilitation Medicine 


\section{INTRODUCTION}

Negative physiologic adaptations, such as impaired hemodynamic response, respiratory dysfunctions, and muscle atrophy are observed in individuals with stroke [1]. These changes can affect the health status and daily activities after stroke. Therefore, assessment of the cardiorespiratory response to exercise is essential for the purpose of determining exercise capacity, training prescription, treatment efficacy evaluation, and/or investigation of exercise-induced adaptations of the oxygen transport/utilization system in individuals with stroke [2].

Transient responses of oxygen uptake $\left(\mathrm{VO}_{2}\right)$ kinetics to a constant-load exercise at an intensity below the ventilatory threshold are classified into three phases. The time constant of $\mathrm{VO}_{2}$ kinetics in phase $2\left(\tau \mathrm{VO}_{2}\right)$ represents the speed of the mono-exponential increase in $\mathrm{VO}_{2}$ toward a steady state level, which reflects the ability of the cardiopulmonary system to deliver and the skeletal muscles to utilize the increased level of oxygen needed for aerobic metabolism [3]. A greater $\tau \mathrm{VO}_{2}$ is associated with poor health status, aging, and physical deconditioning [4-8]. $\tau \mathrm{VO}_{2}$ in individuals with stroke has been reported to be larger than that in healthy adults $[9,10]$, which is caused by an impairment of the oxygen delivery system rather than the mechanism of oxygen utilization [11]. In addition, a larger $\tau \mathrm{VO}_{2}$ is associated with fewer daily walking steps in these individuals [12].

$\mathrm{VO}_{2}$ data during exercise onset are fitted to a monoexponential model to estimate $\tau \mathrm{VO}_{2}$ [4]. Breathing typically has inherent irregularities that can result in breathto-breath fluctuations in gas exchange. Therefore, $\mathrm{VO}_{2}$ data obtained from multiple repeated bouts of exercise are ensemble-averaged to improve the goodness of fit for mono-exponential modelling that is reported as the 95\% confidence interval (CI) for $\tau \mathrm{VO}_{2}$ [13-15]. Although averaging the $\mathrm{VO}_{2}$ transition of at least three exercise bouts has been considered necessary to estimate $\tau \mathrm{VO}_{2}$ $[13,15,16]$, increasing the number of exercise repetitions corresponds to an increase in the burden on participants and in the time required to complete an exercise test. As a single exercise bout can minimize the testing burden, it may be desirable for persons who have difficulty in completing the multiple bouts of exercise. A single bout and three repeated bouts of exercise have been carried out to assess $\mathrm{VO}_{2}$ kinetics in individuals with stroke
$[9,10,12,17]$. However, it has not been reported whether $\tau \mathrm{VO}_{2}$ estimated from a single exercise bout can reflect the relative difference in $\tau \mathrm{VO}_{2}$ obtained from three averaged bouts in individuals with stroke. Previous experimental and simulation studies of healthy adults reported that the estimates of $\tau \mathrm{VO}_{2}$ were not changed [13] or progressively decreased $[15,16]$ as the number of averaged exercise bouts increased from one to three, although the reason for the decrease in $\tau \mathrm{VO}_{2}$ was not determined. As cardiorespiratory responses to exercise differ between individuals with stroke and healthy adults $[9,18,19]$, understanding the influence of averaging multiple exercise bouts on the estimates of $\tau \mathrm{VO}_{2}$ in individuals with stroke will help clinicians working in stroke rehabilitation to determine the number of exercise bouts for estimating the $\tau \mathrm{VO}_{2}$ of their patients.

Hence, the present study aimed to determine the relationship between $\tau \mathrm{VO}_{2}$ estimated from a single bout and that obtained from the average of three exercise bouts in individuals with stroke. We hypothesized that $\tau \mathrm{VO}_{2}$ estimated from a single exercise bout would be strongly correlated with but greater than that obtained from the average of three exercise bouts, considering the findings of previous studies that examined the influence of averaging multiple exercise bouts on $\tau \mathrm{VO}_{2}$ in healthy adults $[13,15,16]$.

\section{MATERIALS AND METHODS}

\section{Study design}

The study protocol was approved by the ethics committees of Tokyo Bay Rehabilitation Hospital (Approval No. 172-2) and Shinshu University (Approval No. 3813). All participants provided written informed consent prior to their enrolment in the study.

\section{Participants}

Participants with stroke were recruited from a subacute rehabilitation ward from January 2018 to March 2020. The diagnosis was confirmed based on computed tomography findings. The inclusion criteria were as follows: (1) age 40-80 years, (2) the first-ever stroke occurred within 180 days, (3) ability to maintain a target cadence of 50 rpm during exercise, and (4) Mini-Mental State Examination score $\geq 24$ [20]. The exclusion criteria were as follows: (1) limited range of motion and/or pain that could affect 
exercise test performance; (2) unstable medical conditions, such as unstable angina, uncontrolled hypertension, or tachycardia; (3) use of beta-blockers; and (4) any comorbid neurological disorders.

\section{Procedure}

On the first day, participants performed a symptomlimited graded exercise test to determine the workload for a submaximal exercise test. Within a week after the symptom-limited graded exercise test, the submaximal constant-load exercise test was performed at approximately $80 \%$ of the workload corresponding to the ventilatory threshold [9].

\section{Exercise testing}

Participants were instructed to refrain from food consumption for 3 hours, caffeine intake for at least 6 hours, and vigorous physical activity for 24 hours prior to the exercise test [21]. Exercise testing was performed on a recumbent cycle ergometer (Strength Ergo 240; Mitsubishi Electric Engineering Co. Ltd., Tokyo, Japan). Participants were instructed to maintain a target cadence of $50 \mathrm{rpm}$ during the symptom-limited graded and submaximal constant-load exercise tests [21].

All participants rested for 5 minutes before measurements were obtained. The symptom-limited graded exercise test started with a warm-up at $10 \mathrm{~W}$ for 3 minutes, followed by an increment of $10 \mathrm{~W}$ every minute [21]. The test was terminated if a participant exhibited signs of angina, dyspnea, inability to maintain a cycling cadence of $\geq 40 \mathrm{rpm}$, hypertension ( $>250 \mathrm{mmHg}$ systolic or $>115$ $\mathrm{mmHg}$ diastolic), or a decrease in systolic blood pressure by $>10 \mathrm{mmHg}$ despite the increase in workload $[22,23]$. Expired gas was measured on a breath-by-breath basis using an expired gas analyzer (Aerosonic AT-1100; ANIMA Corp., Tokyo, Japan). Prior to expired gas data collection, the analyzer was calibrated using gas mixtures with accurately known concentrations of oxygen and carbon dioxide.

The ventilatory threshold was identified using a combination of the following criteria: (1) the point of deflection of carbon dioxide output versus $\mathrm{VO}_{2}$ (V-slope method), (2) the point at which the ventilatory equivalent of oxygen reaches its minimum or starts to increase, without an increase in the ventilatory equivalent of carbon dioxide, and (3) the point at which the end-tidal oxygen fraction reaches its minimum or starts to increase, without a decrease in the end-tidal carbon dioxide fraction [24]. The V-slope method was prioritized when all three criteria yielded different results [25]. The first threshold was determined as the average of values provided by two independent raters (JM and YI), when the difference in $\mathrm{VO}_{2}$ values of the corresponding points, as determined by the two raters, was $<100 \mathrm{~mL} / \mathrm{min}$. In case of any discrepancy, a third experienced rater (KO) judged the point, and the first threshold was taken as the average of the two closest values [26].

The submaximal constant-load exercise test consisted of three 6 -minute periods of pedaling at $80 \%$ of the workload corresponding to the ventilatory threshold, with a rest between each repetition. The first trial of three exercise bouts was preceded by a 3 -minute resting period.

\section{Data fitting}

Occasional errant $\mathrm{VO}_{2}$ values due to coughing, swallowing, or talking were first deleted from the dataset when $\mathrm{VO}_{2}$ exceeded 3 standard deviations of the local mean, defined as the average of 2 subsequent breaths and 2 preceding breaths [14]. Data from individual trials were interpolated on a second-by-second basis and timealigned such that the onset of exercise corresponds to 0 second $[15,27]$. The interpolated data from the first trial of three exercise bouts were used to estimate $\mathrm{VO}_{2}$ kinetics parameters for a single exercise bout. In addition, the interpolated data collected from three exercise bouts were ensemble-averaged to obtain $\mathrm{VO}_{2}$ kinetics parameters for three averaged bouts as the criterion. The first 20 seconds of data after exercise onset was eliminated, as the increase in $\mathrm{VO}_{2}$ during this period merely reflects the increase in pulmonary blood flow rather than changes in tissue gas exchange $[4,15]$. To estimate $\tau \mathrm{VO}_{2}$, nonlinear least squares regression with GraphPad Prism software for Windows version 7.00 (GraphPad Software, San Diego, CA, USA) was applied to the onset phase using monoexponential functions with the following format:

Onset kinetics: $\mathrm{VO}_{2(\mathrm{t})}=$ baseline $\mathrm{VO}_{2}$ value $+\mathrm{A} \times(1-$ $\left.\exp ^{-(\mathrm{t}-\mathrm{TD}) / \tau}\right)$

where $\mathrm{VO}_{2(\mathrm{t})}$ represents $\mathrm{VO}_{2}$ at a given time $(\mathrm{t}) ; \mathrm{A}$, the amplitude of the increase in $\mathrm{VO}_{2}$ above the baseline value; $\mathrm{TD}$, the time delay; and $\tau$, the time constant. The baseline $\mathrm{VO}_{2}$ value was defined as the average $\mathrm{VO}_{2}$ during the last minute of the resting period, whereas the steady-state 
$\mathrm{VO}_{2}$ value referred to the average $\mathrm{VO}_{2}$ during the last minute of exercise. The goodness of fit for mono-exponential modelling was evaluated as the $95 \% \mathrm{CI}$ for $\tau \mathrm{VO}_{2}$. A narrower 95\% CI for $\tau \mathrm{VO}_{2}$ reflects a smaller signal noise $[13,15,27]$.

\section{Statistical analysis}

$G^{*}$ Power software version 3.1.9.2 (Heinrich-Heine-Universitat, Dusseldorf, Germany) was used for sample size calculation [28]. Previous experimental studies of healthy adults compared the $\mathrm{VO}_{2}$ kinetics parameters among different number of averaged exercise bouts in eight to nine participants $[13,16]$. For correlation analysis, as we hypothesized a strong correlation between $\tau \mathrm{VO}_{2}$ estimated from a single exercise bout and that obtained from the average of three exercise bouts, we used an estimated effect size of 0.70 (very large) [29]. In addition, considering a statistical power of 0.95 and an alpha level of 0.05 , the required sample size was estimated at 16 .

Data normality was assessed using the Shapiro-Wilk test, which determined that the assumption of normality was met for the $\mathrm{VO}_{2}$ kinetics parameters ( $\left.\mathrm{p}>0.05\right)$. Pearson product-moment correlation coefficient was used to examine the correlation of the $\mathrm{VO}_{2}$ kinetics parameters, including the baseline $\mathrm{VO}_{2}$ value, the amplitude of the increase in $\mathrm{VO}_{2}$ during onset exercise, time delay, and $\tau \mathrm{VO}_{2}$, between a single bout and three averaged bouts. Correlations were categorized as negligible $(|\mathrm{r}|<0.3)$, low

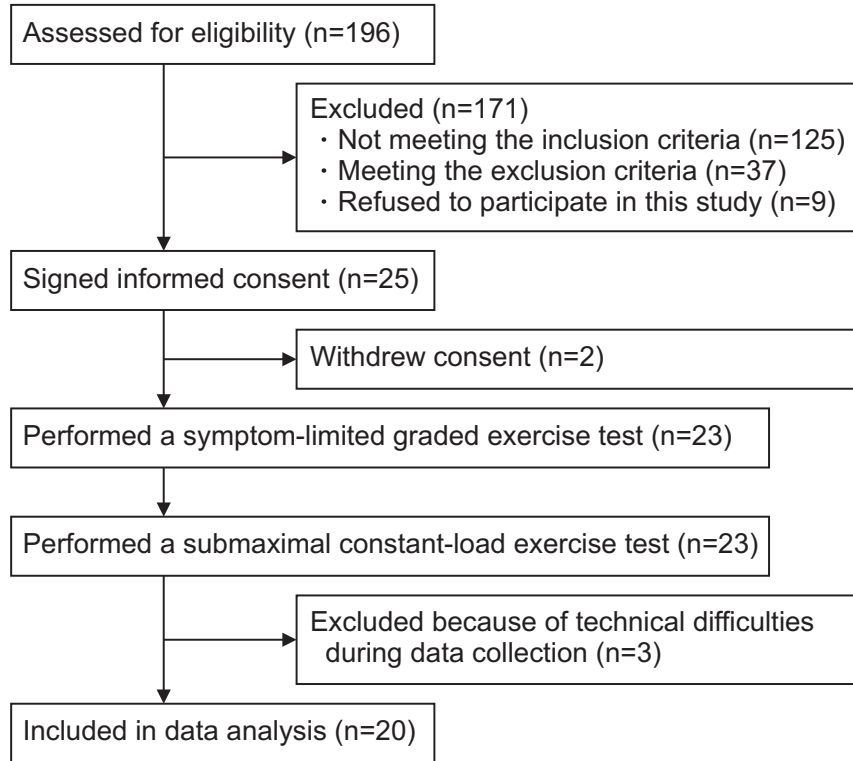

Fig. 1. Flow chart of study participants.
$(0.3 \leq|\mathrm{r}|<5.0)$, moderate $(0.5 \leq|\mathrm{r}|<0.7)$, high $(0.7 \leq|\mathrm{r}|<0.9)$, and very high $(0.9 \leq|\mathrm{r}|)[30]$. When a high to very high correlation of $\tau \mathrm{VO}_{2}$ existed between a single bout and three averaged bouts, we additionally performed a liner regression analysis to obtain an equation that converted $\tau \mathrm{VO}_{2}$ estimated from a single exercise bout to that obtained from the average of three exercise bouts. A paired t-test was used to compare the $\mathrm{VO}_{2}$ kinetics parameters and the 95\% CI for $\tau \mathrm{VO}_{2}$ between different numbers of averaged exercise bouts. We also used one-way repeated measures analysis of variance, followed by a paired t-test with Bonferroni correlation to investigate whether the $\mathrm{VO}_{2}$ kinetics parameters and the $95 \% \mathrm{CI}$ for $\tau \mathrm{VO}_{2}$ changed during repeated trials. Statistical analyses were conducted using GraphPad Prism software for Windows version 7.00 (GraphPad Software, San Diego, CA, USA). Any p-values $<0.05$ were considered to indicate statistical significance.

Table 1. Participants' characteristics

\begin{tabular}{lc}
\hline \multicolumn{1}{c}{ Variable } & Value \\
\hline Age $(\mathrm{yr})$ & $59.5 \pm 10.1$ \\
Sex & 16 \\
\hline Male & 4 \\
\hline Female & $1.67 \pm 0.06$ \\
Height $(\mathrm{m})$ & $61.1 \pm 7.9$ \\
\hline Weight $(\mathrm{kg})$ & $21.9 \pm 2.1$ \\
\hline BMI $\left(\mathrm{kg} / \mathrm{m}^{2}\right)$ & \\
Type of stroke & 10 \\
\hline Ischemic & 10 \\
\hline Hemorrhagic & \\
\hline Side of motor paresis & 11 \\
\hline Right & 9 \\
\hline Left & $70.5 \pm 30.7$ \\
\hline Time since stroke (day) & $28.5 \pm 8.1$ \\
\hline Fugl-Meyer lower extremity motor score & \\
\hline Antihypertensive medications & 3 \\
\hline Angiotensin II receptor blockers & 4 \\
\hline Calcium channel blockers & \\
\hline Comorbidities & 3 \\
\hline Diabetes mellitus & 5 \\
\hline Hyperlipidemia & 7 \\
\hline Hypertension & \\
\hline
\end{tabular}

Values are presented as mean \pm standard deviation or number.

BMI, body mass index. 


\section{RESULTS}

\section{Participants}

A flow chart describing participant enrolment for this study is shown in Fig. 1. A total of 23 participants performed the symptom-limited graded and submaximal constant-load exercise tests. Expired gas during the submaximal constant-load exercise test could not be measured in three participants because of technical difficulties. Consequently, 20 participants were included in the analysis. The participants' characteristics are summarized in Table 1.

\section{Exercise testing}

No serious adverse events occurred during or after the symptom-limited graded and submaximal constant-load exercise tests. All participants had to stop performing the symptom-limited graded exercise test because of their inability to maintain a cycling cadence of $>40 \mathrm{rpm}$. The ventilatory threshold was determined in all participants. The mean $\mathrm{VO}_{2}$ at the ventilatory threshold was $14.7 \pm 2.7$ $\mathrm{mL} / \mathrm{kg} / \mathrm{min}$, and the median work rate corresponding to approximately $80 \%$ of $\mathrm{VO}_{2}$ at the ventilatory threshold was $10.0 \mathrm{~W}$ (interquartile range, 10.0-17.5 W).

The representative $\mathrm{VO}_{2}$ responses during exercise onset obtained from a single bout and three averaged bouts are shown in Fig. 2. In addition, the measurement values of $\mathrm{VO}_{2}$ kinetics parameters and the $95 \% \mathrm{CI}$ for $\tau \mathrm{VO}_{2}$ are presented in Table 2 .

\section{Correlations between $\mathrm{VO}_{2}$ kinetics parameters} estimated from a single bout and the average of three exercise bouts

There was a high to very high correlation between a single bout and three averaged exercise bouts for the baseline $\mathrm{VO}_{2}$ value $(\mathrm{r}=0.853 ; 95 \%$ CI, 0.660-0.941;
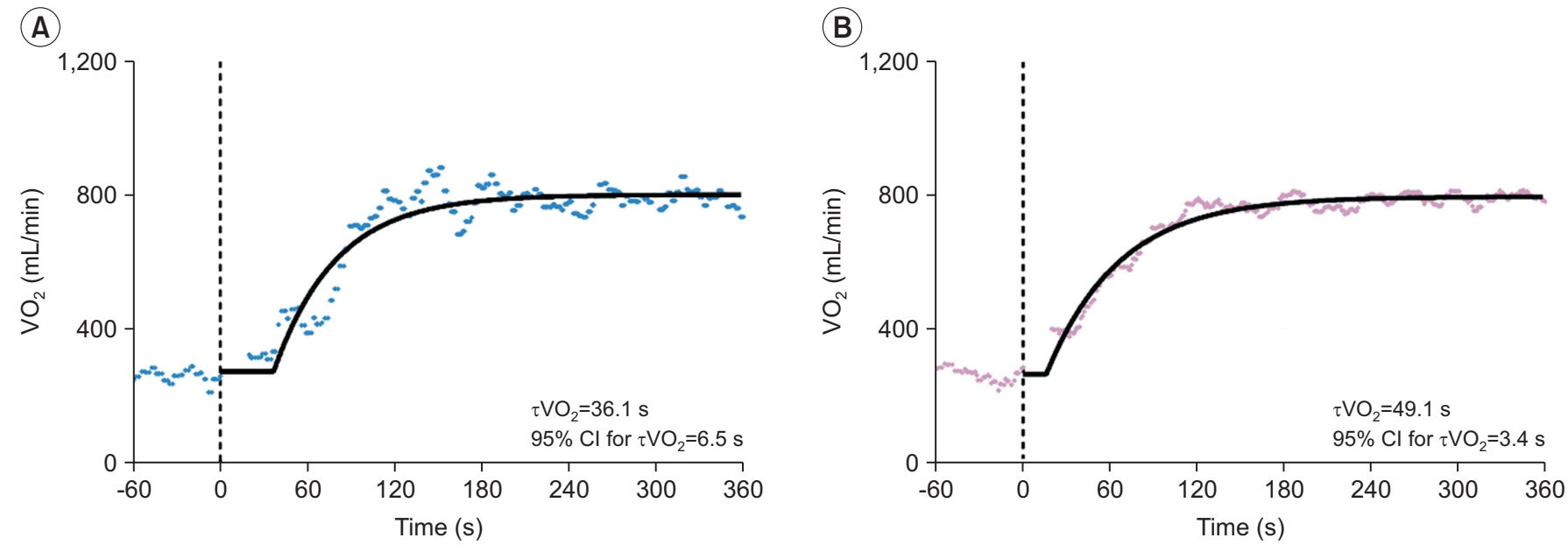

Fig. 2. Typical oxygen uptake kinetics during exercise onset obtained from a single bout (A) and the average of three exercise bouts (B). Model fits on the data are displayed with a solid line. The vertical dashed line indicates the onset of exercise. CI, confidence interval; $\tau \mathrm{VO}_{2}$, time constant of oxygen uptake kinetics; $\mathrm{VO}_{2}$, oxygen uptake.

Table 2. Comparisons of oxygen uptake kinetics parameters and the $95 \%$ CI between a single exercise bout and three averaged exercise bouts

\begin{tabular}{lcccc}
\hline \multicolumn{1}{c}{ Variable } & Single bout & Three averaged bouts & Mean difference (95\% CI) & p-value \\
\hline Baseline $(\mathrm{mL} / \mathrm{min})$ & $263.6 \pm 44.4$ & $268.3 \pm 40.2$ & $-4.7(-15.6$ to 6.2$)$ & 0.381 \\
\hline Amplitude $(\mathrm{mL} / \mathrm{min})$ & $567.7 \pm 141.0$ & $567.2 \pm 148.3$ & $0.5(-16.2$ to 15.3$)$ & 0.954 \\
Time delay $(\mathrm{s})$ & $18.8 \pm 10.3$ & $16.9 \pm 7.3$ & $2.5(-0.6$ to 5.5$)$ & 0.107 \\
$\tau \mathrm{VO}_{2}$ (s) & $34.8 \pm 14.0$ & $38.5 \pm 13.4$ & $-3.7(-6.2$ to -1.2$)$ & 0.006 \\
$95 \%$ CI for $\tau \mathrm{VO}_{2}$ (s) & $6.0 \pm 2.2$ & $4.1 \pm 1.2$ & $2.0(1.1$ to 2.8$)$ & $<0.001$ \\
\hline
\end{tabular}

Values are presented as mean \pm standard deviation.

$\mathrm{CI}$, confidence interval; $\tau \mathrm{VO}_{2}$, time constant of oxygen uptake kinetics. 

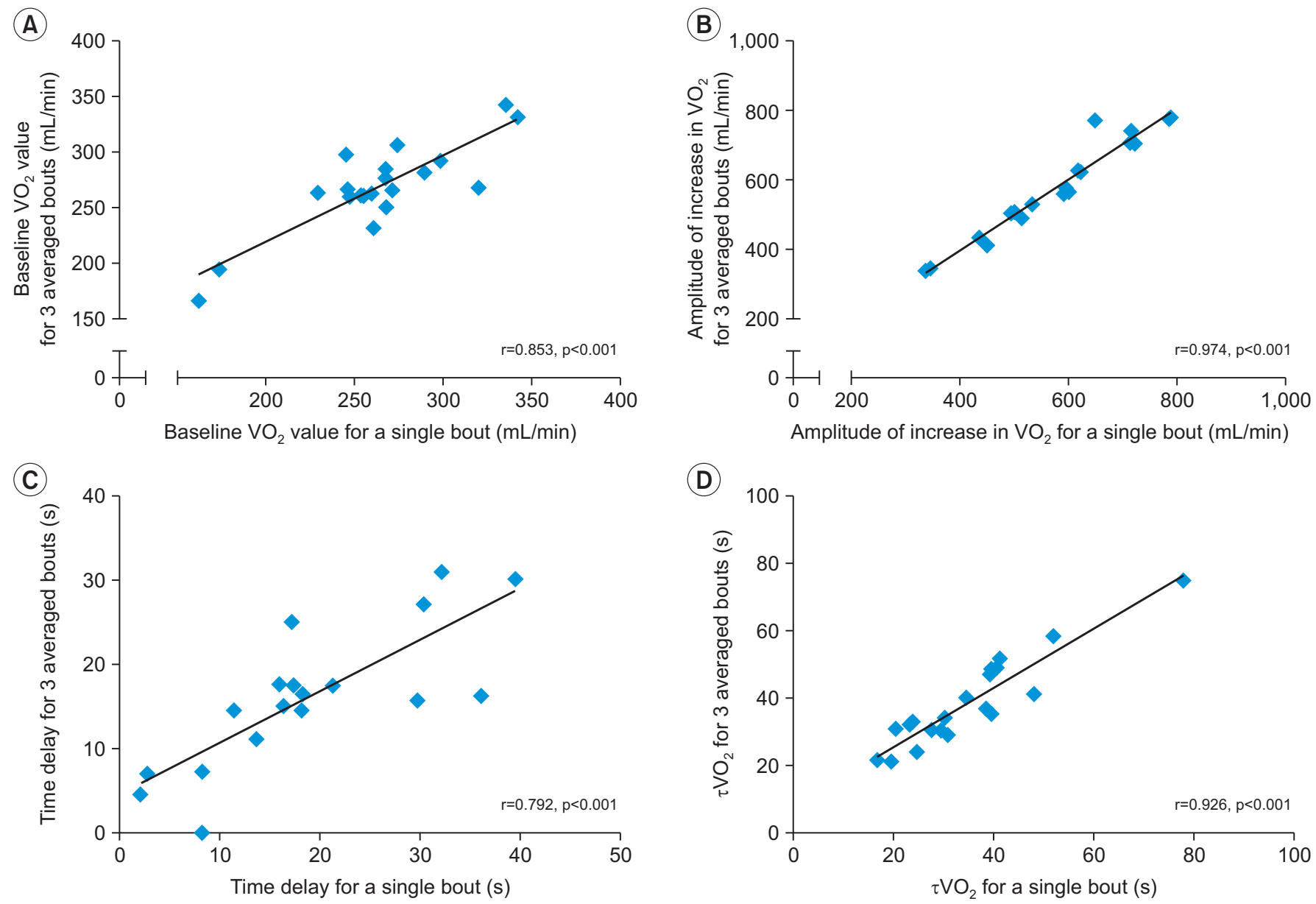

Fig. 3. Correlations between a single bout and three averaged exercise bouts for the baseline $\mathrm{VO}_{2}$ value (A), the amplitude of increase in $\mathrm{VO}_{2}(\mathrm{~B})$, the time delay (C), and $\tau \mathrm{VO}_{2}(\mathrm{D})$. A high to very high correlation is shown between a single bout and three averaged exercise bouts for all $\mathrm{VO}_{2}$ kinetics parameters $(\mathrm{p}<0.001)$. A linear regression model (D) to convert $\tau \mathrm{VO}_{2}$ estimated from a single exercise bout to that obtained from the average of three exercise bouts is as follows: $\tau \mathrm{VO}_{2}$ for three averaged bouts $=0.887 \times \tau \mathrm{VO}_{2}$ for a single bout $+7.618\left(\mathrm{R}^{2}=0.857, \mathrm{p}<0.001\right) . \tau \mathrm{VO}{ }_{2}$, time constant of oxygen uptake kinetics; $\mathrm{VO}_{2}$, oxygen uptake.

$\mathrm{p}<0.001$ ) (Fig. 3A), the amplitude of the increase in $\mathrm{VO}_{2}$ $(\mathrm{r}=0.974 ; 95 \% \mathrm{CI}, 0.935-0.990 ; \mathrm{p}<0.001)$ (Fig. 3B), the time delay ( $\mathrm{r}=0.792 ; 95 \% \mathrm{CI}, 0.537-0.914 ; \mathrm{p}<0.001$ ) (Fig. 3C), and $\tau \mathrm{VO}_{2}(\mathrm{r}=0.931 ; 95 \% \mathrm{CI}, 0.831-0.973 ; \mathrm{p}<0.001)$ (Fig. $3 \mathrm{D})$. The linear regression analysis revealed the following equation to convert $\tau \mathrm{VO}_{2}$ estimated from a single exercise bout to that obtained from the average of three exercise bouts: $\tau \mathrm{VO}_{2}$ for three averaged bouts $=0.887 \times \tau \mathrm{VO}_{2}$ for a single bout $+7.618\left(\mathrm{R}^{2}=0.857, \mathrm{p}<0.001\right)$.

\section{Differences between a single bout and three averaged} exercise bouts for $\mathrm{VO}_{2}$ kinetics parameters and the $95 \%$ $\mathrm{Cl}$ for $\tau \mathrm{VO}_{2}$

There were no significant differences in the baseline
$\mathrm{VO}_{2}$ value, the amplitude of the increase in $\mathrm{VO}_{2}$, and time delay between a single bout and three averaged exercise bouts ( $p>0.05)$. However, $\tau \mathrm{VO}_{2}$ estimated from a single exercise bout was significantly smaller than that obtained from the average of three exercise bouts (mean difference $=-3.7 ; 95 \% \mathrm{CI},-6.2$ to $-1.2 ; \mathrm{p}=0.006$ ). By contrast, the $95 \% \mathrm{CI}$ for $\tau \mathrm{VO}_{2}$ for a single exercise bout was significantly greater than that for three averaged exercise bouts (mean difference $=2.0 ; 95 \% \mathrm{CI}, 1.1-2.8 ; \mathrm{p}<0.001$ ) (Table 2).

\section{Comparisons in $\mathrm{VO}_{2}$ kinetics parameters and the $95 \%$}

$\mathrm{Cl}$ for $\tau \mathrm{VO}_{2}$ between the first, second, and third trials of the three exercise bouts

There were no significant differences in the baseline 
(A)

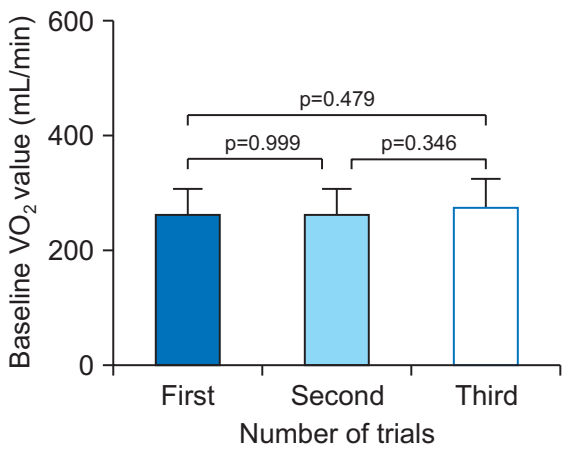

(D)

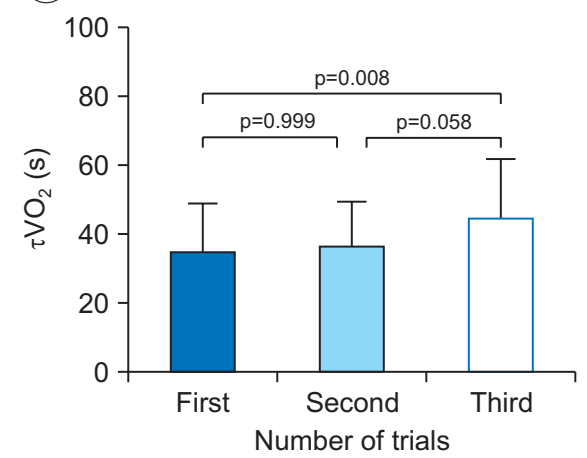

(B)

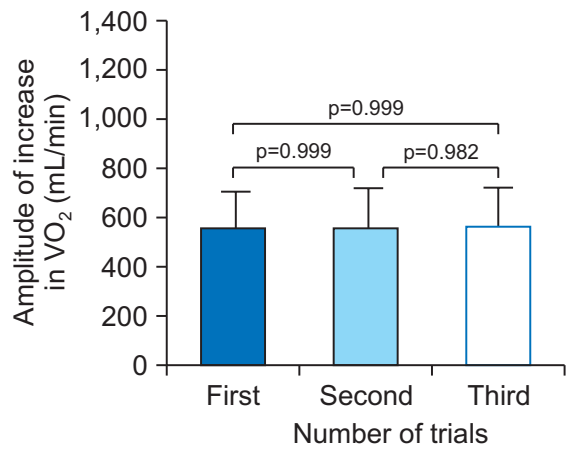

(E)

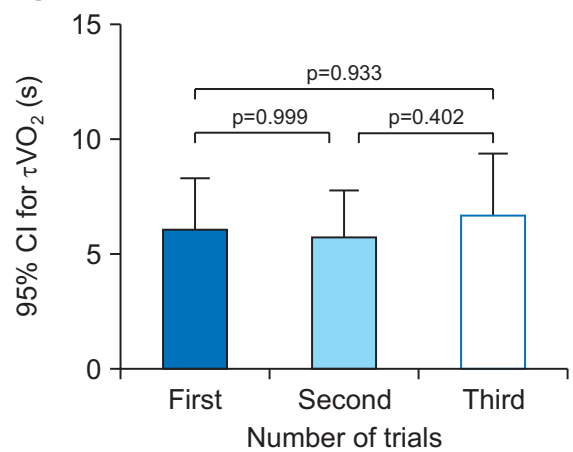

(C)

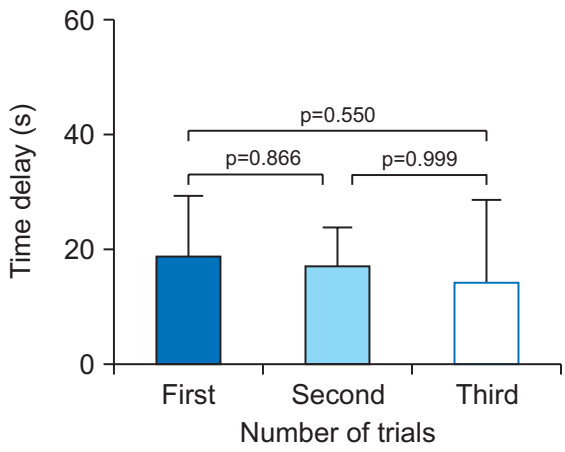

Fig. 4. Comparisons of the baseline $\mathrm{VO}_{2}$ value (A), the amplitude of increase in $\mathrm{VO}_{2}(\mathrm{~B})$, the time delay (C), $\tau \mathrm{VO}_{2}(\mathrm{D})$, and $95 \% \mathrm{CI}$ for $\tau \mathrm{VO}_{2}$ (E). $\tau \mathrm{VO}_{2}$ estimated from the first trial is significantly smaller than that obtained from the third trial ( $\mathrm{p}=0.008$ ). $\mathrm{CI}$, confidence interval; $\tau \mathrm{VO}_{2}$, time constant of oxygen uptake kinetics; $\mathrm{VO}_{2}$, oxygen uptake.

$\mathrm{VO}_{2}$ value (Fig. 4A), the amplitude of the increase in $\mathrm{VO}_{2}$ (Fig. 4B), the time delay (Fig. 4C), and the $95 \% \mathrm{CI}$ for $\tau \mathrm{VO}_{2}$ (Fig. 4E) among the trials ( $\mathrm{p}>0.05)$. However, $\tau \mathrm{VO}_{2}$ estimated from the first trial was significantly smaller than that obtained from the third trial of the three exercise repetitions (mean difference $=-9.7$; $95 \% \mathrm{CI},-17.0$ to -2.4 ; $\mathrm{p}=0.008$ ) (Fig. 4D).

\section{DISCUSSION}

To the best of our knowledge, this is the first study to investigate the relationships between $\mathrm{VO}_{2}$ kinetics parameters estimated from a single bout and those obtained from the average of multiple exercise bouts in individuals with stroke. We found a very strong relationship between $\tau \mathrm{VO}_{2}$ estimated from a single exercise bout and that obtained from the average of three exercise bouts in individuals with stroke. Although there were no significant differences in the baseline $\mathrm{VO}_{2}$ value, the amplitude of increase in $\mathrm{VO}_{2}$, and time delay between a single bout and three averaged exercise bouts, the $\tau \mathrm{VO}_{2}$ for a single exercise bout was underestimated compared to that obtained by averaging three exercise bouts. In line with previous studies $[14,15]$, the $95 \% \mathrm{CI}$ for $\tau \mathrm{VO}_{2}$ decreased by increasing the number of averaged exercise bouts from one to three. Nevertheless, increasing the number of exercise repetitions corresponds to an increase in the burden on participants. Therefore, $\tau \mathrm{VO}_{2}$ estimated from a single exercise bout may be a useful parameter in the assessment of $\mathrm{VO}_{2}$ kinetics during exercise onset in individuals who are unable to perform multiple bouts of exercise. Our findings might help clinicians working in stroke rehabilitation to determine the number of exercise bouts for estimating $\tau \mathrm{VO}_{2}$ of their patients.

The mean $\tau \mathrm{VO}_{2}$ obtained from the average of three exercise bouts was similar to the data of previous studies in individuals with stroke $[9,17]$. Although the noise around the mean $\mathrm{VO}_{2}$ response from a single exercise bout was larger than that from three bouts averaged, the results of the correlation analyses suggest that $\tau \mathrm{VO}_{2}$ for a single exercise bout can reflect the relative difference in $\tau \mathrm{VO}_{2}$ for three averaged exercise bouts. The mean dif- 
ference between $\tau \mathrm{VO}_{2}$ estimated from a single exercise bout and that obtained from three exercise bouts averaged was -3.7 seconds. Tomczak et al. [9] found that the $\tau \mathrm{VO}_{2}$ was significantly greater in patients with stroke than in age-, sex-, and activity-matched healthy adults with a mean difference of approximately 10 seconds. In addition, Murakami et al. [17] reported that the mean change in $\tau \mathrm{VO}_{2}$ from baseline to the end of the 8-week program of exercise was 6.2 seconds in people with stroke. Benson et al. [15] proposed that a minimally important difference for a significant change in $\tau \mathrm{VO}_{2}$ during interventional and comparative studies is 5 seconds. Thus, although the mean difference in $\tau \mathrm{VO}_{2}$ between a single and three averaged exercise bouts observed in this study was smaller than the minimally important difference for $\tau \mathrm{VO}_{2}$, we note that $\tau \mathrm{VO}_{2}$ from a single exercise bout may be underestimated compared to that obtained by averaging three exercise bouts in individuals with stroke. The linear regression model obtained in this study may allow the conversion of $\tau \mathrm{VO}_{2}$ estimated from a single exercise bout to that obtained from the average of three exercise bouts. Further studies with a larger sample size are warranted to confirm the robustness of the linear regression equation.

A smaller $\tau \mathrm{VO}_{2}$ for a single exercise bout than that for three averaged exercise bouts may be attributed to a smaller $\tau \mathrm{VO}_{2}$ estimated from the first trial than that obtained from the third trial of the three exercise repetitions, because the baseline $\mathrm{VO}_{2}$ value, the amplitude of the increase in $\mathrm{VO}_{2}$, the time delay, and the $95 \% \mathrm{CI}$ for $\tau \mathrm{VO}_{2}$ were not significantly different among the exercise trials. No studies have reported an increase in $\tau \mathrm{VO}_{2}$ during exercise repetitions in healthy adults. Thus, the results of the present study may be specific to individuals with stroke. However, our data could not reveal the reason for the statistically significant difference between $\tau \mathrm{VO}_{2}$ for the first and third trials. Various physiological mechanisms, such as cardiovascular function, oxygen utilization by skeletal muscles, and autonomic nervous system, potentially affect the increase in $\mathrm{VO}_{2}$ during exercise onset $[5,11,31]$. The assessment of these mechanisms may help to elucidate the reason for the increase in $\tau \mathrm{VO}_{2}$ during exercise repetitions observed in this study.

This study has several limitations. First, our participants were recruited from a subacute rehabilitation ward, which may limit the ability to generalize the results to people with acute and chronic stroke. Second, this study had no control group. The difference in $\tau \mathrm{VO}_{2}$ among different numbers of averaged exercise bouts has been reported only in healthy young adults $[13,15,16]$. Therefore, further research including both individuals with stroke and age-matched healthy controls is needed to ascertain whether the findings of this study are specific to individuals with stroke. Third, this study did not compare the test-retest reliability of $\tau \mathrm{VO}_{2}$ with and without multipletrial averaging; further studies investigating this point are necessary. Finally, only the mode of cycling was selected for $\mathrm{VO}_{2}$ kinetic assessment, as previous studies assessing $\mathrm{VO}_{2}$ kinetics in individuals with stroke used a cycle ergometer $[9,10,12,17]$. However, a treadmill, total-body recumbent stepper, robotics-assisted tilt table, or arm crank ergometer has been used for exercise testing in individuals with stroke $[21,26,32]$. Hence, the generalization of our findings to other modes of exercise should be made with caution.

In conclusion, the results of the correlation analysis suggest that $\tau \mathrm{VO}_{2}$ for a single exercise bout could reflect the relative difference in $\tau \mathrm{VO}_{2}$ for three averaged exercise bouts in individuals with stroke. Therefore, a single exercise bout may be desirable for assessing $\mathrm{VO}_{2}$ kinetics in individuals who have difficulty in completing multiple bouts of exercise. However, clinicians should note that $\tau \mathrm{VO}_{2}$ estimated from a single exercise bout might be underestimated compared to $\tau \mathrm{VO}_{2}$ obtained by averaging three exercise bouts in determining the number of exercise bouts for estimating $\tau \mathrm{VO}_{2}$ in their patients with stroke.

\section{CONFLICT OF INTEREST}

No potential conflict of interest relevant to this article was reported.

\section{ACKNOWLEDGMENTS}

This work was supported by a grant from the Funds for a Grant-in-Aid for Young Scientists to Kazuaki Oyake (No. 18K17730). The funding source had no role in the study design; in the collection, analysis, and interpretation of the data; in writing the report; and in the decision to submit the article for publication. 


\section{AUTHOR CONTRIBUTION}

Conceptualization: Oyake K, Otaka Y, Momose K. Methodology: all authors. Formal analysis: Oyake K, Otaka Y, Momose K. Funding acquisition: Oyake K. Project administration: Oyake K, Baba Y, Momose K. Visualization: Oyake K, Otaka Y, Momose K. Writing-original draft: Oyake K. Writing-review and editing: Oyake K, Otaka Y, Momose K. Approval of final manuscript: all authors.

\section{REFERENCES}

1. Billinger SA, Coughenour E, Mackay-Lyons MJ, Ivey FM. Reduced cardiorespiratory fitness after stroke: biological consequences and exercise-induced adaptations. Stroke Res Treat 2012;2012:959120.

2. Wittink H, Verschuren O, Terwee C, de Groot J, Kwakkel G, van de Port I. Measurement properties of maximal cardiopulmonary exercise tests protocols in persons after stroke: a systematic review. J Rehabil Med 2017;49:689-99.

3. Arena R, Humphrey R, Peberdy MA. Measurement of oxygen consumption on-kinetics during exercise: implications for patients with heart failure. J Card Fail 2001;7:302-10.

4. Poole DC, Jones AM. Oxygen uptake kinetics. Compr Physiol 2012;2:933-96.

5. Kemps HM, Schep G, Zonderland ML, Thijssen EJ, De Vries WR, Wessels B, et al. Are oxygen uptake kinetics in chronic heart failure limited by oxygen delivery or oxygen utilization? Int J Cardiol 2010;142:138-44.

6. Alexander NB, Dengel DR, Olson RJ, Krajewski KM. Oxygen-uptake (VO2) kinetics and functional mobility performance in impaired older adults. J Gerontol A Biol Sci Med Sci 2003;58:734-9.

7. Hummel SL, Herald J, Alpert C, Gretebeck KA, Champoux WS, Dengel DR, et al. Submaximal oxygen uptake kinetics, functional mobility, and physical activity in older adults with heart failure and reduced ejection fraction. J Geriatr Cardiol 2016;13:450-7.

8. George MA, McLay KM, Doyle-Baker PK, Reimer RA, Murias JM. Fitness level and not aging per se, determines the oxygen uptake kinetics response. Front Physiol 2018;9:277.

9. Tomczak CR, Jelani A, Haennel RG, Haykowsky MJ, Welsh R, Manns PJ. Cardiac reserve and pulmonary gas exchange kinetics in patients with stroke. Stroke 2008;39:3102-6.

10. Manns PJ, Tomczak CR, Jelani A, Haennel RG. Oxygen uptake kinetics: associations with ambulatory activity and physical functional performance in stroke survivors. J Rehabil Med 2010;42:259-64.

11. Oyake K, Baba Y, Suda Y, Murayama J, Mochida A, Kondo K, et al. Cardiorespiratory mechanisms underlying the impaired oxygen uptake kinetics at exercise onset after stroke. Ann Phys Rehabil Med 2021;64:101465.

12. Katoh J, Murakami M, Hirayama M, Nagata Y, Hayakawa M, Tanizaki T. Correlation of pedometric measurement of daily physical activity with exercise endurance by oxygen uptake kinetics in ambulatory stroke patients. J Phys Ther Sci 2002;14:77-80.

13. Spencer MD, Murias JM, Lamb HP, Kowalchuk JM, Paterson DH. Are the parameters of VO2, heart rate and muscle deoxygenation kinetics affected by serial moderate-intensity exercise transitions in a single day? Eur J Appl Physiol 2011;111:591-600.

14. Lamarra N, Whipp BJ, Ward SA, Wasserman K. Effect of interbreath fluctuations on characterizing exercise gas exchange kinetics. J Appl Physiol (1985) 1987;62:2003-12.

15. Benson AP, Bowen TS, Ferguson C, Murgatroyd SR, Rossiter HB. Data collection, handling, and fitting strategies to optimize accuracy and precision of oxygen uptake kinetics estimation from breath-by-breath measurements. J Appl Physiol (1985) 2017;123:227-42.

16. McNulty CR, Robergs RA. Repeat trial and breath averaging: recommendations for research of $\mathrm{VO} 2$ kinetics of exercise transitions to steady-state. Mov Sport Sci 2019;(106):37-44.

17. Murakami M, Katoh J, Hirayama M, Hayakawa M, Tanizaki T, Furukawa H. Physical fitness and exercise endurance measured by oxygen uptake kinetics in stroke patients. J Phys Ther Sci 2002;14:73-6.

18. Jakovljevic DG, Moore SA, Tan LB, Rochester L, Ford GA, Trenell MI. Discrepancy between cardiac and physical functional reserves in stroke. Stroke 2012;43:1422-5.

19. Sisante JF, Mattlage AE, Arena R, Rippee MA, Billinger SA. Decreased tidal volume may limit cardiopulmonary performance during exercise in subacute stroke. J Cardiopulm Rehabil Prev 2015;35:334-41. 
20. Folstein MF, Folstein SE, McHugh PR. "Mini-mental state": a practical method for grading the cognitive state of patients for the clinician. J Psychiatr Res 1975;12:189-98.

21. van de Port IG, Kwakkel G, Wittink H. Systematic review of cardiopulmonary exercise testing post stroke: Are we adhering to practice recommendations? J Rehabil Med 2015;47:881-900.

22. Oyake K, Baba Y, Ito N, Suda Y, Murayama J, Mochida A, et al. Cardiorespiratory factors related to the increase in oxygen consumption during exercise in individuals with stroke. PLoS One 2019;14:e0217453.

23. Fletcher GF, Ades PA, Kligfield P, Arena R, Balady GJ, Bittner VA, et al. Exercise standards for testing and training: a scientific statement from the American Heart Association. Circulation 2013;128:873-934.

24. Binder RK, Wonisch M, Corra U, Cohen-Solal A, Vanhees L, Saner H, et al. Methodological approach to the first and second lactate threshold in incremental cardiopulmonary exercise testing. Eur J Cardiovasc Prev Rehabil 2008;15:726-34.

25. Bosch PR, Holzapfel S, Traustadottir T. Feasibility of measuring ventilatory threshold in adults with strokeinduced hemiparesis: implications for exercise prescription. Arch Phys Med Rehabil 2015;96:1779-84.

26. Saengsuwan J, Berger L, Schuster-Amft C, Nef T, Hunt $\mathrm{KJ}$. Test-retest reliability and four-week changes in cardiopulmonary fitness in stroke patients: evaluation using a robotics-assisted tilt table. BMC Neurol 2016;16:163.

27. Keir DA, Murias JM, Paterson DH, Kowalchuk JM. Breath-by-breath pulmonary $\mathrm{O} 2$ uptake kinetics: effect of data processing on confidence in estimating model parameters. Exp Physiol 2014;99:1511-22.

28. Erdfelder E, Faul F, Buchner A. GPOWER: a general power analysis program. Behav Res Methods Instrum Comput 1996;28:1-11.

29. Maher JM, Markey JC, Ebert-May D. The other half of the story: effect size analysis in quantitative research. CBE Life Sci Educ 2013;12:345-51.

30. Mukaka MM. Statistics corner: a guide to appropriate use of correlation coefficient in medical research. Malawi Med J 2012;24:69-71.

31. Capelli C, Adami A, Antonutto G, Cautero M, Tam E. Oxygen deficits and oxygen delivery kinetics during submaximal intensity exercise in humans after 14 days of head-down tilt-bed rest. Eur J Appl Physiol 2009;107:51-9.

32. Oyake K, Yamaguchi T, Oda C, Kudo D, Kondo K, Otaka Y, et al. Unilateral arm crank exercise test for assessing cardiorespiratory fitness in individuals with hemiparetic stroke. Biomed Res Int 2017;2017:6862041. 